\title{
Discrimination of human cytotoxic lymphocytes from regulatory and B-lymphocytes by orthogonal light scattering
}

\author{
L.W.M.M. Terstappen ${ }^{1}$, B.G. De Grooth ${ }^{1}$, C.H.H. Ten Napel ${ }^{2}$, W. Van Berkel ${ }^{2}$ and J. Greve ${ }^{1}$ \\ 'Twente University of Technology, Cell Characterization Group, Department of Applied Physics, P.O. Box 217, 7500 AE Enschede, \\ and $^{2}$ Ziekenzorg Hospital, Department of Internal Medicine, Haakshergerstraat 55, 7513 ER Enschede, The Netherlands
}

(Received 20 March 1986, revised received 2 September 1986, accepted 3 September 1986)

Light scattering properties of human lymphocyte subpopulations selected by immunofluorescence were studied with a flow cytometer. Regulatory and B-lymphocytes showed a low orthogonal light scatter signal, whereas cytotoxic lymphocytes identified with leu-7, leu-11 and leu-15 revealed a large orthogonal light scatter signal. Two populations in light scatter histograms could be observed with monoclonal antibodies directed against determinants present on both regulatory and cytotoxic lymphocytes. By analysis of the lymphocytes of 16 individuals we found a linear relation between the number of cells with a large orthogonal light scattering and the number of cytotoxic lymphocytes identified with leu-7, leu-11 and $l e u-15$. These observations demonstrate physical differences between cytotoxic lymphocytes and regulatory and $\mathrm{B}$ lymphocytes. Moreover, the results suggest a method to estimate the amount of cytotoxic lymphocytes without using monoclonal antibodies.

Key words: Flow cytometry; Orthogonal light scattering; Cytotoxic lymphocyte

\section{Introduction}

Light scattering can be used to discriminate between different cell types. Forward light scattering is related to cell size (Mullaney et al., 1970; Visser et al., 1980), whereas orthogonal light scattering is determined by internal and external cell structure (Brunsting et al., 1974; Van den Engh et al., 1981; Benson et al., 1984). This is frequently used in flow cytometry (FCM) to select lymphocytes, monocytes, or granulocytes in a sample of human leukocytes. Recently we have shown that T8-positive lymphocytes can be sep-

Correspondence to: L.W.M.M. Terstappen, Twente University of Technology, Cell Characterization Group, Department of Applied Physics, P.O. Box 217, 7500 AE Enschede, The Netherlands.

Abbreviations: Mabs, monoclonal antibodies; FCM, flow cytometer. arated into two populations, one with relatively low and one with a relatively high orthogonal light scatter signal (Terstappen et al., 1986). Our results indicated that these T8-positive populations exist of suppressor cells and cytotoxic cells, respectively.

The importance of human cytotoxic lymphocytes in the defence against viral infections and neoplasms is shown in an increasing number of studies (Yap et al., 1978; Talmadge et al., 1980; Herberman and Orthaldo, 1981; Engers et al., 1982; Lukacher et al., 1984; Moller et al., 1985). Enumeration of the total amount of human cytotoxic lymphocytes is impossible; only subpopulations of cytotoxic lymphocytes can be identified through recognition by monoclonal antibodies. In this paper we have investigated whether cytotoxic lymphocytes can be identified on basis of their orthogonal light scattering properties. 


\section{Materials and methods}

\section{Preparation of lymphocytes}

Human blood was collected by venipuncture into vacutainer tubes containing heparin as anticoagulant (150 USP U sodium heparin $/ 10 \mathrm{ml}$, Venoject Terumo Europe). Lymphocytes were obtained after density separation as described in detail elsewhere (Terstappen et al., 1986). Lymphocytes were adjusted to a concentration of $1 \times$ $10^{7}$ lymphocytes per $\mathrm{ml}$ with a standard buffer (PBS containing $0.05 \%$ sodium azide and 1\% bovine serum albumin (BSA)).

\section{Immunofluorescence}

The following monoclonal antibodies (Mabs) were used: purified Mabs, T8 (anti-leu-2a, from Becton Dickinson, Amersfoort, The Netherlands) T suppressor-cytotoxic marker, T4 (RIV 6, from National Institute for Public Health and Milieu (RIVM), Rijswijk, The Netherlands) T helper-inducer marker; biotin-conjugated Mab, B1 (from Coulter Electronics, Hoekloos, Schiedam, The Netherlands) pan B-cell marker; fluorescein (FITC)-labeled Mabs, T3 (anti-leu-4, from Becton Dickinson) pan T-cell marker, leu-8 (anti-leu-8 from Becton Dickinson) T helper, suppressor and monocyte marker, leu-7 and leu-11 (anti-leu-7, anti-leu-11, from Becton Dickinson) identifying subpopulations of natural killer, killer, and large granular lymphocytes (Abo et al., 1982; Lanier et al., 1983); phycoerythrine (PE)-labeled Mabs, leu15 (anti-CR3 (anti-leu-15)), from Becton Dickinson) identifying a major part of cytotoxic lymphocytes, monocytes and granulocytes, leu-1 (anti-leu-1, from Becton Dickinson) pan T-cell marker and a minority of B-lymphocytes. For second step reagents FITC-conjugated goat antimouse immunoglobulin (FITC GAM) (Central Laboratory of Blood Transfusion Service (CLB) Amsterdam, The Netherlands) and FITC-conjugated avidin (from Becton Dickinson) were used. All reagents were pretitered by using normal lymphocytes to determine optimal conditions for binding. $100 \mu \mathrm{l}$ cell suspension was incubated with $20 \mu 1$ diluted Mab. Mabs were diluted in standard buffer for $30 \mathrm{~min}$ at room temperature. For indirect fluorescence and two color fluorescence the cells were washed twice with standard buffer and resuspended in $100 \mu \mathrm{l}$ diluted second step reagent, or $20 \mu 1$ diluted Mab, respectively. The cells were incubated for $30 \mathrm{~min}$ at room temperature. After incubation the cells were washed twice and resuspended in $1 \mathrm{ml}$ standard buffer and kept on ice until use.

A 'polyclonal' antiserum was made by incubating the lymphocytes simultaneously with the Mabs leu-7, leu-11 and leu-15 at the same time in an equal dilution as when used separately.

Flow cytometric analysis was performed the same day to preserve optimal binding of monoclonal antibody and light scatter properties of lymphocytes (Nicholson et al., 1984).

\section{Flow cytometric (FCM) measurements}

The FCM was built to our own design as described in detail elsewhere (Terstappen et al., 1986). A $3 \mathrm{~W}$ argon ion laser tuned at $488 \mathrm{~nm}$ operating at a light intensity of $100 \mathrm{~mW}$ was used. FITC emission was measured with a six-cavity bandfilter (510-550 nm, Pomfret Research Optics, Stanford, CT). The PE emission was measured with an OG 590 colored glass filter (Schott, Tiel, The Netherlands). For two color fluorescence measurements a $550 \mathrm{~nm}$ dichroic mirror $103 \mathrm{SW}$ P017, Melles Griot, Irvine, CA, placed at an angle of $45^{\circ}$ ) was used to separate the FITC from the PE emission. Measurements with the 'polyclonal' antiserum were performed by using an OG 530 and KV 520 colored glass filters (Schott). Reproducible conditions for the measurement of light scatter histograms of lymphocytes were obtained by adjustment of the FCM with microspheres of the same batch. Light scatter histograms of lymphocyte subpopulations were measured after gating on the fluorescence intensity of immunofluorescently labeled cells. Gates are chosen such that cells are definitely positive: less than $0.1 \%$ of the cells of control samples fall within the gates.

The cut-off level for determining the percentage of positive cells was chosen at such a level that less than $1 \%$ of the cells were positive with the control samples. The results of measurements of at least 10000 cells are displayed in the figures.

\section{Results}

Pronounced differences in light scatter properties were observed when different lymphocyte sub- 
populations were analyzed. The main differences were observed in the orthogonal light scattering.

Fig. 1 shows flow cytometric histograms of orthogonal light scattering of all lymphocytes (Fig. 1a), leu-8-positive (leu-8 ${ }^{+}$, Fig. 1b), leu-7-positive (leu- $7^{+}$, Fig. 1c) and B1-positive (B1 ${ }^{+}$, Fig. 1d) lymphocytes. From the figures it is evident that the orthogonal light scatter histogram of all lymphocytes (Fig. 1a) reveals at least two subpopulations. The majority of helper and suppressor lymphocytes (identified with leu-8) and B-
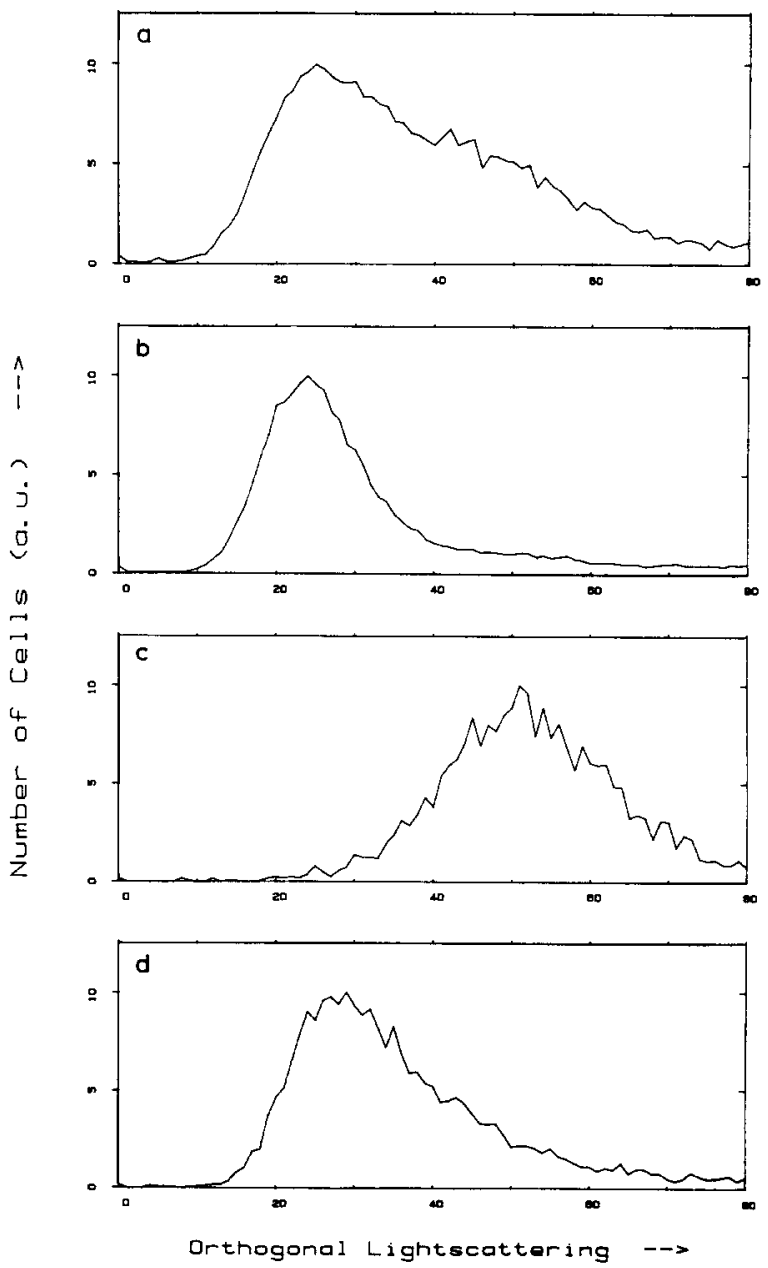

Fig. 1. Orthogonal light scatter histograms of all lymphocytes (a), leu- $8^{+}$lymphocytes (b), leu- $7^{+}$lymphocytes (c) and B1 ${ }^{+}$ lymphocytes $(d)$, respectively. The peak position of leu- $8^{+}(b)$ and $\mathrm{B1}^{+}(d)$ lymphocytes corresponds with the first peak in the histogram of all lymphocytes, whereas the peak position of the leu- $7^{+}$(c) lymphocytes correspond with the second peak in the histogram of all lymphocytes $(a)$. lymphocytes (identified with B1) have a low orthogonal light scattering whereas the cytotoxic lymphocytes identified with leu-7 have a large orthogonal light scatter signal. For comparison the relative magnitude of the mean orthogonal light scattering signal of regulatory and B-lymphocytes, cytotoxic lymphocytes, monocytes and granulocytes is $1.0,1.6,4.0$ and 11 , respectively.

Based on this observed difference in orthogonal light scattering of $l e u-8^{+}$and $\mathrm{B1}^{+}$versus $l e u-7^{+}$ lymphocytes measured histograms of all lymphocytes were divided into two components $\mathrm{A}$ and $\mathrm{B}$. The percentage of each population was estimated by counting the number of cells in it as is indicated in Fig. 2. With ten different donors we studied the contribution of lymphocyte subpopulations to each region. For each individual $80-95 \%$ of the leu- $8^{+}$and $\mathrm{T}^{+}$(helper-induced marker) lymphocytes and $80-85 \%$ of $\mathrm{B} 1{ }^{+}$lymphocytes are within region $\mathrm{A}$, whereas more than $95 \%$ of the leu $-7^{+}$, leu- $11^{+}$and leu- $15^{+}$lymphocytes (cytotoxic lymphocytes) lie within region $\mathrm{B}$. In agreement with this, two populations in orthogonal light scatter histograms could be observed with

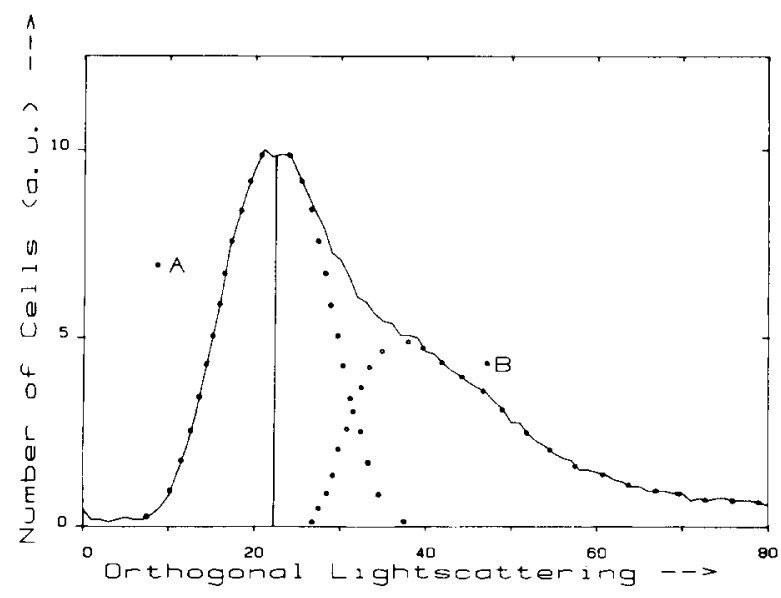

Fig. 2. Orthogonal light scatter histogram of all lymphocytes of a typical healthy donor. The histogram is divided into two components $A$ and $B$, the maximum of the peak position of the component with a low orthogonal light scattering (A) is that from $l e u-8^{+}$and $\mathrm{T}^{+}$orthogonal light scatter histograms. Component $\mathrm{A}$ is obtained by assuming that the population of cells with a low orthogonal light scattering yield a symmetrically shaped histogram. By subtracting this component from the measured histogram the second component (B) was obtained. The number of cells under each curve was counted. 
other monoclonal antibodies directed against determinants present on both regulatory and cytotoxic lymphocytes. Examples are leu-4, identifying the majority of T-lymphocytes, leu-1, identifying the majority of T-lymphocytes and a minor population of B-lymphocytes and T8 identifying suppressor and cytotoxic lymphocytes. These results clearly show that at least the majority of cytotoxic lymphocytes can be distinguished from regulatory and B-lymphocytes on basis of their light scattering properties. The fact that this discrimination is not complete (e.g., 15-20\% of the B-lymphocytes are still found in region $B$ ) can be due to a small overlap of the distributions of the populations. For $l e u-8^{+}$and $\mathrm{T}^{+}$lymphocytes however a relatively large interdonor variation is observed for the percentage of cells found in region B $(5-20 \%)$, suggesting a different cause. By performing two color immunofluorescence flow cytometry we found the existence of lymphocytes exhibiting determinants for both leu-8 and leu-15 (Fig. 3a). In the orthogonal light scatter histogram not only the majority of leu-8 $8^{-}$-leu-15 $15^{+}$lymphocytes have a large orthogonal light scatter signal (Fig. $3 b$ ), but also the leu- $8^{+}$leu- $15^{+}$lymphocytes (fig. $3 c$ ). (Note the cluster on the edge of Fig. $3 c$ which is due to monocytes which are known to be positive with both monoclonal antibodies). On the other hand, the majority of leu- $8^{+}$leu- $15^{-}$lymphocytes have a low orthogonal light scatter signal (Fig. 3d). In order to obtain a marker which identifies the majority of cytotoxic lymphocytes we have made a 'polyclonal' antiserum by incubating the lymphocytes with fluorescently labeled leu-7, leu-11 and leu-15 at the same time. Fig. 4 presents semithree-dimensional plots of the orthogonal light scattering versus the fluorescence of a control sample (Fig. 4a), lymphocytes incubated with leu7, leu-11 and leu-15 (Fig. 4b) and lymphocytes incubated with $\mathrm{T} 4$ (Fig. $4 c$ ). The figures clearly show the two populations with different orthogonal light scatter signal. Almost all lymphocytes with a large orthogonal light scattering are positive with at least one of the Mabs leu-7, leu-11 and leu-15, whereas a great part of the lymphocytes with a low orthogonal light scattering are T4 positive. The relation between large orthogonal light scattering and cytotoxic lymphocytes was tested in the following experiment (Fig. 5). For 16
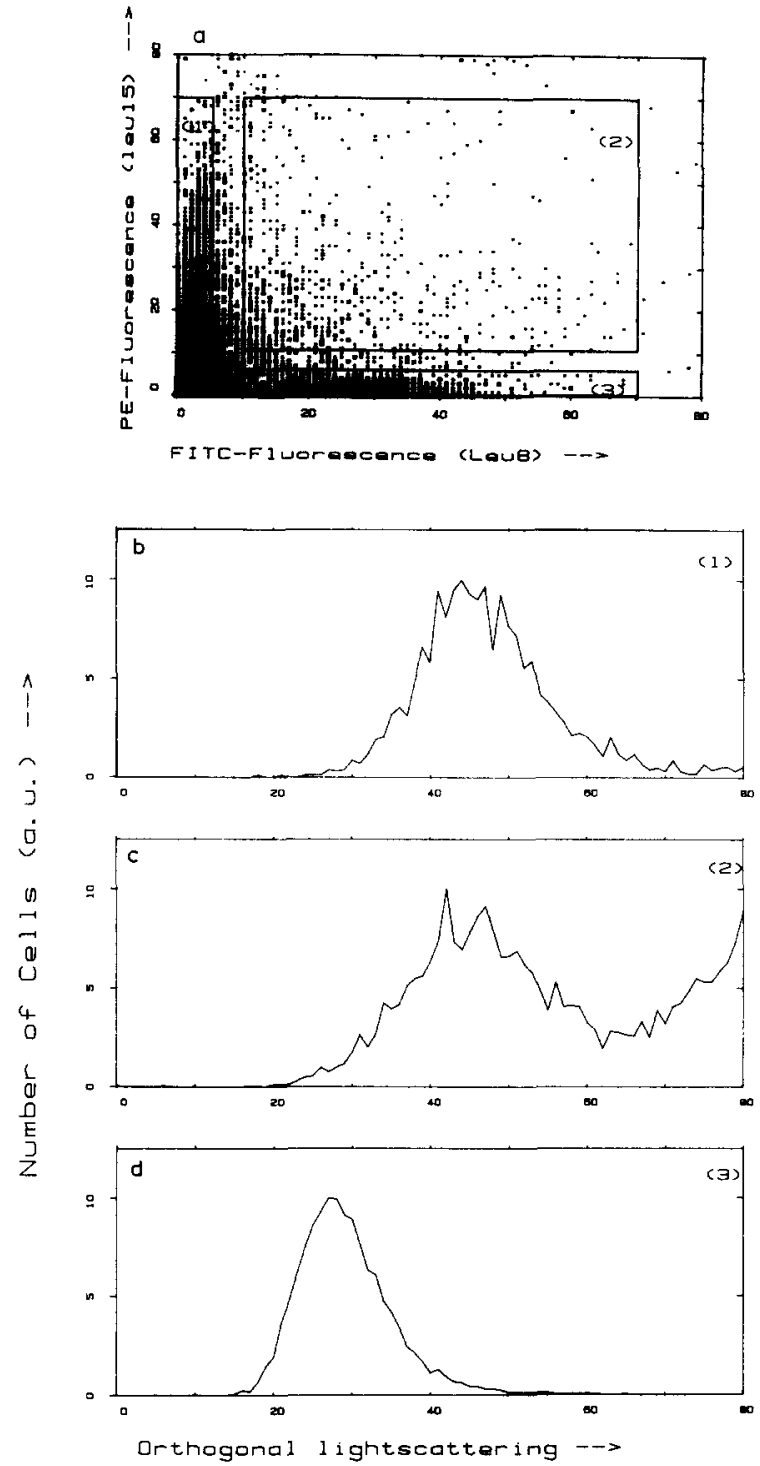

Fig. 3. a: Two color immunofluorescence density map of FITC-labeled anti-leu 8 and phycoerythrine-labeled anti-leu-15 of a healthy individual. Gates on $l e u-8^{-} l e u-15^{+}$(1), leu- $8^{+} l e u-$ $15^{+}(2)$ and $l e u-8^{+} l e u-15^{-}$(3) populations are indicated. $b, c$, $d$ : Orthogonal light scatter histograms of the gated subpopulations leu- $8^{-} l e u-15^{+}$, leu- $8^{+} l e u-15^{+}$, leu- $8^{+} l e u-15^{-}$, respectively.

donors the percentage of cells which are positive with at least one of the monoclonal antibodies leu-7, leu-11 and leu-15 is plotted against the percentage of lymphocytes with a large orthogonal light scattering (Fig. 5). The figure reveals a positive relation between these parameters. The best 

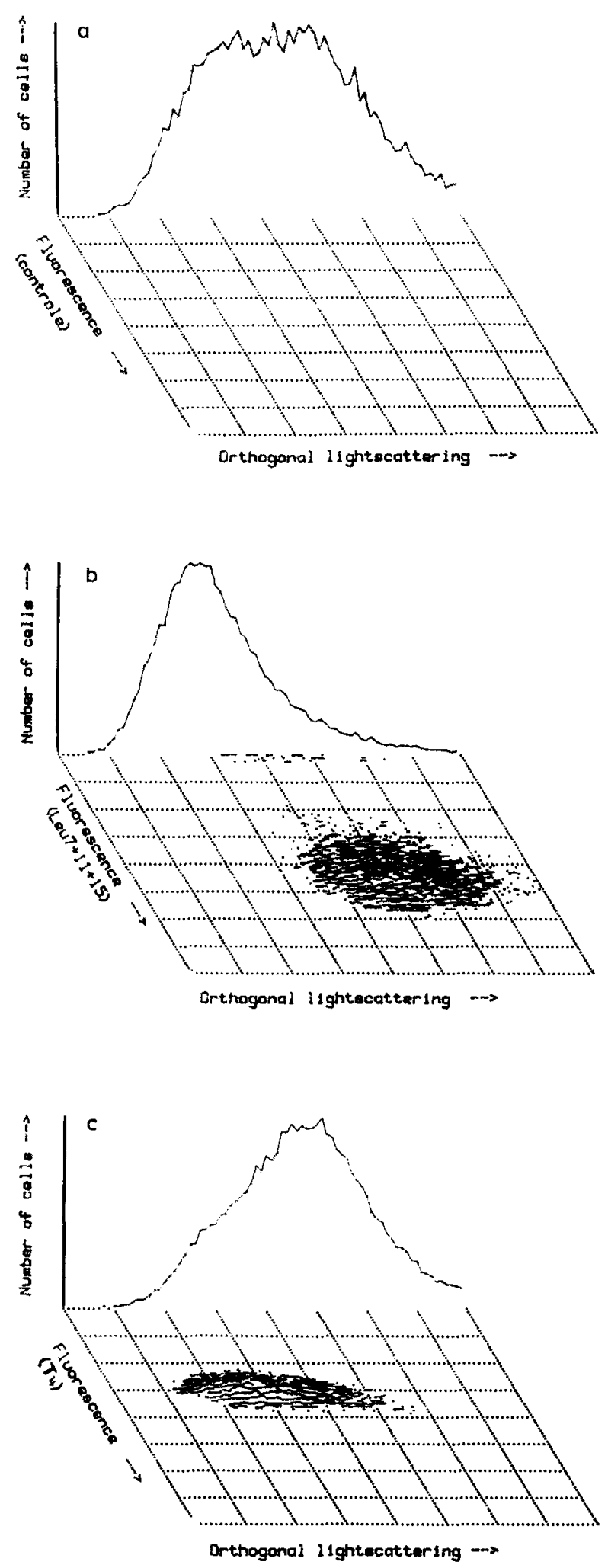

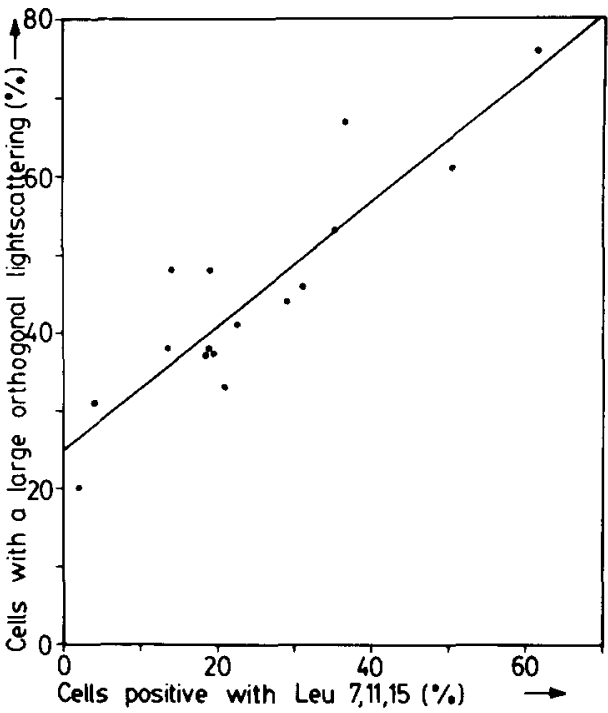

Fig. 5. For 16 donors the percentage of cells which are positive with at least one of the monoclonal antibodies leu-7, leu-11 and leu-15 is plotted against the percentage of lymphocytes with a large orthogonal light scattering. The best linear fit is plotted in the figure.

linear fit has a slope of 0.81 and a correlation coefficient of $r^{2}=0.90$.

Interestingly, the relation between the percentage of lymphocytes with a large orthogonal light scattering and the percentage of lymphocytes positive with each monoclonal antibody separately was less evident.

\section{Discussion}

With orthogonal light scattering granulocytes, monocytes and lymphocytes can be separated. We have demonstrated in an earlier report that with orthogonal light scattering two populations of T8-positive lymphocytes could be distinguished (Terstappen et al., 1986). In that study the hypothesis was raised that the T8-positive lymphocytes with a large orthogonal light scattering were cytotoxic. The results shown in the present study

Fig. 4. Semi-three-dimensional representation of the orthogonal light scattering and fluorescence intensity of lymphocytes. $a$ : Control sample of unstained lymphocytes: all cells appear on the horizontal axis. $b$ : Lymphocytes incubated with the polyclonal antiserum leu-7, leu-11 and leu-15. c. Lymphocytes incubated with T4. 
are in agreement with this hypothesis and extend it to all lymphocytes with a large orthogonal light scattering. The majority of regulatory and B lymphocytes have a low orthogonal light scatter signal whereas cytotoxic lymphocyte subpopulations identified with leu-7, leu-11 and leu-15 have a large orthogonal light scatter signal (Fig. 1). In agreement with this two populations in orthogonal light scatter histograms could be observed with monoclonal antibodies directed against determinants present on both regulatory and cytotoxic lymphocytes.

The results given in Fig. 3 show that the presence of cytotoxic determinants on lymphocytes is correlated with a relatively large orthogonal light scattering. B lymphocytes and 'truly' regulatory lymphocytes (i.e., regulatory lymphocytes without cytotoxic determinants, leu- $8^{+} l e u-15^{-}$) reveal a low orthogonal light scattering.

Strong indications that the reverse of our hypothesis is also true, i.e., lymphocytes with a large orthogonal light scattering are cytotoxic lymphocytes, are obtained from the results shown in Figs. 4 and 5. In Fig. 4 one can see that almost all cells with a large orthogonal light scattering are positive with at least one of the cytotoxic markers used. Furthermore with 16 healthy donors we found a good correlation between the number of cells with a large orthogonal light scattering and the number of cells positive with one of the cytotoxic markers. Not all cytotoxic lymphocytes can be identified with these markers. This explains that the best linear fit found in Fig. 5 does not cross the origin and that there are still some cells with a large orthogonal light scattering which are not positive with our cytotoxic markers (Fig. $4 b$ ). Moreover if the hypothesis is correct we can conclude that on the average $20 \%$ of the cytotoxic cells lack determinants of leu-7, leu-11 and leu-15. A definite confirmation of this hypothesis awaits the availability of antibodies which identify all cytotoxic lymphocytes. Functional tests and morphological studies of both populations isolated by cell sorting are currently being done in our laboratory.

Our observations suggest a method to estimate the amount of cytotoxic lymphocytes without using monoclonal antibodies. This can be done by determining the number of lymphocytes with a large orthogonal light scattering. An interesting possibility is to separate the lymphocytes on account of their light scattering properties with a cell sorter. Functional studies of strongly enriched cytotoxic or regulatory and B-lymphocytes are then possible without blockage of the cell receptors with antibodies. In this way the profound effects which antibody binding may have, on cell function (Kranz et al., 1984; Acuto and Reinherz, 1985; Leeuwenberg et al., 1985; Schmidt et al., 1985; Spits et al., 1985) are prevented.

\section{Acknowledgements}

L.W.M.M. Terstappen and B.G. De Grooth thank J.G.W. Pijls and I. Van der Staal for stimulating support during measurements.

\section{References}

Abo, T., M.D. Cooper and C.M. Balch, 1982, J. Immunol. 129, 1752.

Acuto, O. and E.L. Reinherz, 1985, N. Engl. J. Med. 312, 1100.

Benson, M.C., D.C. McDougal and D.S. Coffey, 1984, Cytometry 5,515 .

Brunsting, A. and P.F. Mullaney, 1974, Biophys. J. 14, 439.

Engers, H.D., A.L. Glasebrook and G.D.J. Sorenson, 1982 , Exp. Med. 156, 1280.

Herberman, R.B. and J.R. Ortaldo, 1981, Science 214, 24.

Kranz, D.M., S. Tonegawa and H.N. Eisen, 1984, Proc. Natl. Acad. Sci. U.S.A. 81, 7922.

Lanier, L.L., A. My Le, J.H. Phillips, N.L. Warner and G.F. Babcock, 1983, J. Immunol. 131, 1789.

Leeuwenberg, J.F.M., H. Spits, W.J.M. Tax and P.J.A. Capel, 1985, Immunol. 134, 3770.

Lukacher, A.E., V.L. Braciale and T.J. Braciale, 1984, Exp. Med. 160, 814.

Moller, J.R., B.R. Zisman, P.C. Quan, A. Schattner, D. Panush, J.K. Rose and B.R. Bloom, 1985, Proc. Natl. Acad. Sci. U.S.A. $82,2456$.

Mullaney, P.F. and P.N. Dean, 1970, Biophys. J. 10, 764.

Nicholson, J.K.A., D.M. Jones, G.D. Cross and J.S. Mcdougal, 1984, J. Immunol. Methods 73, 29.

Schmidt, R.E., R.P. Mac Dermott, G. Bartly, M. Bertovich, D.A. Amato, K.F. Augsten, S.F. Schlossman, R.L. Stevens and J. Ritz, 1985, Nature 318, 289.

Spits, H., H. IJssel, A. Voordouw and J.E. De Vries, 1985, J. Immunol. 134, 2294.

Talmadge, J.E., K.M. Meyers, D.J. Prieur and J.R. Starkey, 1980, Nature 284, 622.

Terstappen, L.W.M.M., B.G. De Grooth, G.M.J. Nolten, C.H.H. Ten Napel, W. Van Berkel and J. Greve, 1986, Cytometry 7, 178

Van den Engh, G., J. Visser and B. Trask, 1981, In: Experimental Haematology Today, eds. S.J. Braun and G.P. Ledney (Springer Verlag, New York) p. 19.

Visser, J.W., G.J. Van den Engh and D.W. Van Bekkum, 1980, Blood Cells 6, 391

Yap, K.L., G.L. Ada and I.F.C. McKenzie, 1978, Nature 273, 238 